\title{
Developmental neurogenetics of sexual dimorphism in Aedes aegypti
}

\author{
Molly Duman-Scheel ${ }^{1,2,3 *}$ and Zainulabeuddin Syed ${ }^{2,3 *}$ \\ ${ }^{1}$ Department of Medical and Molecular Genetics, Indiana University School of Medicine, South Bend, IN, USA, ${ }^{2}$ Eck Institute \\ for Global Health, University of Notre Dame, Notre Dame, IN, USA, ${ }^{3}$ Department of Biological Sciences, University of Notre \\ Dame, Notre Dame, IN, USA
}

OPEN ACCESS

Edited by:

Teun Dekker,

Swedish University of Agricultural

Sciences, Sweden

Reviewed by:

Junwei Jerry Zhu,

United States Department of

Agriculture - Agricultural Research

Service, USA

Christine Merlin,

Texas A\&M University, USA

*Correspondence:

Molly Duman-Scheel,

Department of Medical and Molecular

Genetics, Indiana University School of Medicine, Raclin-Carmichael Hall,

1234 Notre Dame Avenue, South

Bend, IN 46617, USA

mschee/@nd.edu;

Zainulabeuddin Syed,

Department of Biological Sciences,

Galvin Life Sciences Center, University

of Notre Dame, 319, Galvin, Notre

Dame, IN 46556, USA

zsyed@nd.edu

Specialty section:

This article was submitted to

Chemical Ecology,

a section of the journal

Frontiers in Ecology and Evolution

Received: 26 February 2015

Accepted: 02 June 2015

Published: 16 June 2015

Citation:

Duman-Scheel M and Syed Z (2015)

Developmental neurogenetics of sexual dimorphism in Aedes aegypti.

Front. Ecol. Evol. 3:61

doi: 10.3389/fevo.2015.00061
Sexual dimorphism, a poorly understood but crucial aspect of vector mosquito biology, encompasses sex-specific physical, physiological, and behavioral traits related to mosquito reproduction. The study of mosquito sexual dimorphism has largely focused on analysis of the differences between adult female and male mosquitoes, particularly with respect to sex-specific behaviors related to disease transmission. However, sexually dimorphic behaviors are the products of differential gene expression that initiates during development and therefore must also be studied during development. Recent technical advancements are facilitating functional genetic studies in the dengue vector Aedes aegypti, an emerging model for mosquito development. These methodologies, many of which could be extended to other non-model insect species, are facilitating analysis of the development of sexual dimorphism in neural tissues, particularly the olfactory system. These studies are providing insight into the neurodevelopmental genetic basis for sexual dimorphism in vector mosquitoes.

Keywords: mosquito, nanoparticle, siRNA, brain, olfaction, doublesex, development, gene targeting

\section{Sexual Dimorphism, a Critical Aspect of Pathogen Transmission by Vector Mosquitoes}

Mosquitoes, including Aedes aegypti, which exhibits innate sexually dimorphic behaviors that contribute to the transmission of dengue, yellow fever, and chikungunya viruses, are excellent subjects for studies that examine the biological basis of sexual dimorphism. Genes that contribute to mosquito sexual dimorphism, including the development of neural circuitries that promote human host-seeking, female blood-feeding behavior, mating, and oviposition, may represent targets for vector control (Clemons et al., 2010a; Tomchaney et al., 2014). Unfortunately, knowledge concerning the extent of sexual dimorphisms in the structure of the central nervous system (CNS), the control of sex-specific behaviors by sexually dimorphic neurons, and the developmental genetic basis for sexually dimorphic behaviors is limited in all organisms, including insects (Kimura, 2011).

Research on the neurodevelopmental genetic basis for insect sexual dimorphism has largely been restricted to Drosophila melanogaster, a genetically-tractable-albeit highly derived-dipteran insect that displays innate sexually dimorphic behaviors. Although early studies suggested that few significant anatomical sexual dimorphisms exist in the D. melanogaster CNS, more

Abbreviations: CNS, Central nervous system; DETs, differentially expressed transcripts; dsx, doublesex; siRNAs, small interfering RNAs; dsRNA, double-stranded RNA; ORNs, olfactory receptor neurons; OR, olfactory receptor; IRs, ionotropic receptors; TALENs, TALE nucleases; FAIRE-seq, formaldehyde-assisted isolation of regulatory elements paired with DNA sequencing. 
recent investigations indicate that the Drosophila CNS has sexually distinct morphologies that originate during development (reviewed by Kimura, 2011). The availability of molecular markers and transgenic reporters to label particular Drosophila neurons greatly facilitated detection of sex-specific developmental differences. Sex-specific differences likely exist in the developing nervous systems of many other insects. However, given the lack of molecular markers for developing neurons in non-model species, comparable analyses have not yet been performed in most insects.

Mosquito genome projects (Holt et al., 2002; Nene et al., 2007; Arensburger et al., 2010; Neafsey et al., 2015) facilitated research in new facets of mosquito biology, including functional developmental genetics. Magnusson et al. (2011) assessed sex-specific transcriptomes throughout Anopheles gambiae development and characterized the functions of several testisand ovary-specific genes during gonad development. Functional genetic analysis of nervous system development has been performed in A. aegypti (Clemons et al., 2011; Haugen et al., 2011; Mysore et al., 2013, 2014a,b; Sarro et al., 2013), an emerging model for vector mosquito development studies (Clemons et al., 2010a). A recent functional genetic study explored the development of sexual dimorphism in the $A$. aegypti pupal nervous system (Tomchaney et al., 2014). Here, we review these findings and highlight possible future strategies and methodologies for dissecting the developmental neurogenetic basis for sexual dimorphism in A. aegypti, many of which may be applicable to other non-model arthropods.

\section{Global and Spatial Analysis of Sexually Dimorphic Gene Expression in the Developing A. aegypti Nervous System}

Custom microarrays were used to examine global differences in female vs. male gene expression in the developing $A$. aegypti pupal head (Tomchaney et al., 2014). Head tissues were prepared $24 \mathrm{~h}$ after puparium formation, a critical period for nervous system development (Mysore et al., 2011, 2013, 2014a,b). At this time point, which follows periods of extensive proliferative activity and pupal histolysis, neuropils characteristic of the adult brain, including the antennal lobe, central complex, and optic lobe neuropils, have begun to form. Extensive neural process outgrowth, targeting of higher order brain neurons, synapse formation, and arborization also occur, and the increased neuropil density of the adult is generated (Mysore et al., 2011). In total, 2527 differentially expressed transcripts (DETs) were identified. Analysis of DETs indicated that dimorphic expression of genes linked to proteolysis, metabolism, catabolic and biosynthetic processes, ion transport, cell growth, and proliferation underlie differences in developing A. aegypti males and females.

Sex-specific pupal brain spatial expression patterns were assessed for a subset of DETs (Figure 1; Tomchaney et al., 2014). These investigations were facilitated by the work of Mysore et al. (2011), who used cross-reactive Drosophila antibodies to establish the first set of molecular markers for the developing mosquito brain. Many of the antibodies work well in conjunction with a combined whole mount in situ hybridization/protein localization protocol (Haugen et al., 2010), which employs a detergent-treatment permeabilization step that has facilitated mRNA localization in many arthropod species (Patel et al., 2001; Duman-Scheel et al., 2002). The results obtained validated the microarray data and laid a foundation for future studies. For example, differential expression of the growth regulators cyclin-dependent kinase 4/6 (cdk4/6) and p53 (Figures 1C,I) may contribute to sexually dimorphic neurite outgrowth (Di Giovanni et al., 2006; Flannery et al., 2010). p53 also controls apoptosis (reviewed by Sutcliffe et al., 2003), suggesting that this process may be regulated in a sex-specific manner in the developing brain. Differential expression of synaptojanin (synj) (Figure 1G), which regulates endocytosis at the Drosophila synapse (Verstreken et al., 2003), was also detected in $A$. aegypti. Furthermore, geko, which mediates Drosophila olfactory responses to ethanol (Shiraiwa et al., 2000) and is dimorphically expressed in $A$. aegypti (Figure 1E), is an interesting target for future functional studies. These expression studies, which detected sex-specific gene expression in the optic lobe, antennal lobe, and mushroom body (Figure 1; also confirmed in sectioned brains), suggested that sex-specific differences exist in the visual and olfactory systems and the processing of sensory information and invoked the question of how dimorphic gene expression is regulated in the developing mosquito nervous system.

\section{Doublesex, a Regulator of Sex-Specific Gene Expression in the Developing Mosquito Brain}

The D. melanogaster doublesex ( $d s x$ ) gene encodes a key terminal transcription factor in the sex-determination pathway (Kimura et al., 2005; Mellert et al., 2010). Drosophila $d s x$ pre-mRNAs are spliced in a sex-specific manner (Burtis and Baker, 1989; Ryner et al., 1996), generating male (DsxM) and female (DsxF) proteins with a common $\mathrm{N}$-terminus and DNA-binding domain, but distinct C-termini that differentially direct sex-specific gene expression (Christiansen et al., 2002; Camara et al., 2008). Male and female $d s x$ splice variants were detected in A. aegypti (Salvemini et al., 2011), and analysis of their expression (Tomchaney et al., 2014) revealed sexually dimorphic $d s x$ expression patterns in the A. aegypti antennal lobe and mushroom body (Figure 2). These sex-specific expression patterns differ from $D$. melanogaster, in which sexually dimorphic $d s x$ expression was detected in only small subsets of neurons (Lee et al., 2002; Rideout et al., 2010). Moreover, $d s x$ is expressed much more broadly in the $A$. aegypti female and male pupal brain. For example, $d s x$ expression is not detected in the $D$. melanogaster pupal optic lobe, but sex-specific isoforms of $d s x$ are expressed abundantly in A. aegypti pupal optic lobes (Figure 2). These results suggest that Dsx may play a more prominent role in the regulation of sex-specific neural development in $A$. aegypti. Furthermore, search of the $A$. aegypti genome sequence uncovered 732 Dsx consensus binding sites, most of which are associated with genes that group under gene ontology 


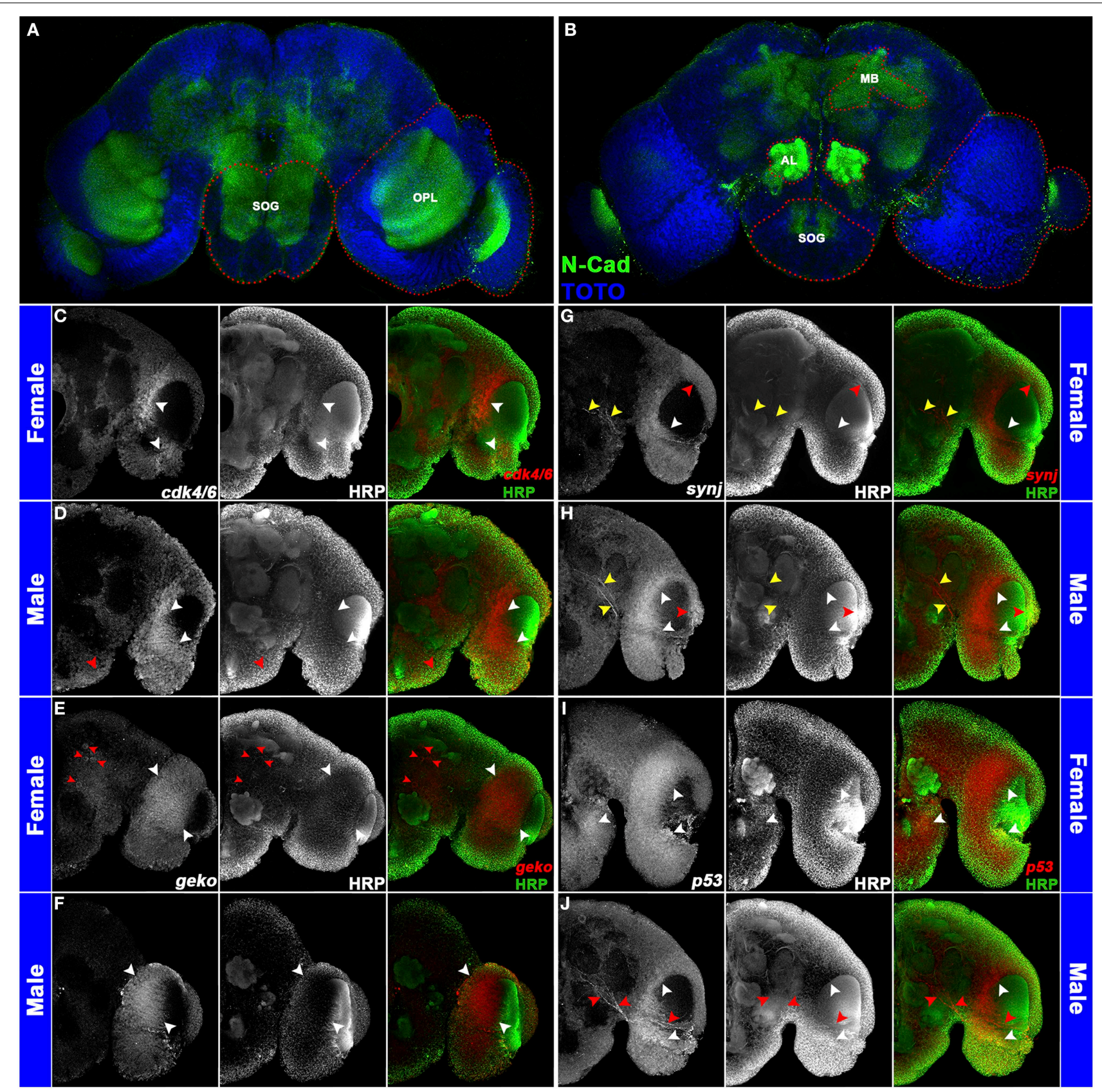

FIGURE 1 | Sex-specific gene expression in the A. aegypti pupal brain. The antennal lobe (AL), optic lobe (OPL), suboesophageal ganglion (SOG), and mushroom body (MB) regions are marked (red dots) in a whole brain labeled with anti-N-Cadherin (green in $\mathbf{A}, \mathbf{B}$ ) and TOTO nuclear stain (blue in $\mathbf{A}, \mathbf{B}$ ). These regions were assessed through confocal imaging following whole mount in situ hybridization and anti-HRP staining (C-J). Five merged Z-stacks (totaling 5 microns) of brain hemisegments (oriented dorsal upward in $\mathbf{C}-\mathbf{J}$ ) labeled through anti-HRP staining (center panels in $\mathbf{C}-\mathbf{J}$; green in overlays at right) and riboprobes corresponding to the indicated transcripts (left panels in $\mathbf{C}-\mathbf{J}$; red in overlays at right) are displayed. Differential expression of $c d k 4 / 6$ (C,D), geko (E,F), synj $(\mathbf{G}, \mathbf{H})$, and p53 $(\mathbf{I}, \mathbf{J})$ is shown in $24 \mathrm{~h}$ pupal brains of

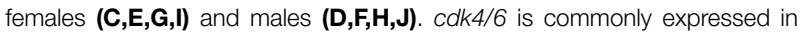
the optic lobe in both sexes (white arrowheads in $\mathbf{C , D}$ ), but additional cdk $4 / 6$ expression is detected in the ventral suboesophageal ganglion of males (red arrowhead in D). geko, which is expressed in the optic lobe of both sexes (white arrowheads in $\mathbf{E , F}$ ), is expressed in additional large cell bodies near the female midbrain and in the female antennal lobe (red arrowheads in E). Expression of synj is detected in the optic lobe (white/red arrowheads in $\mathbf{G}, \mathbf{H}$ ) and in a subset of midbrain neurons (yellow arrowheads in $\mathbf{G}, \mathbf{H}$ ). Sex-specific synj expression is detected in the optic lobe (red arrowheads in $\mathbf{G}, \mathbf{H}$ ), and midbrain levels of synj are generally higher in males (compare expression adjacent to yellow arrowheads in G,H). p53 expression is detected in the suboesophageal ganglion and optic lobe of females (white arrowheads in I). p53 expression is also detected in the male optic lobe (white arrowheads in $\mathbf{J}$ ), but not in the subesophageal ganglion of males. Male-specific p53-expressing neurons are found adjacent to the antennal lobe (red arrowheads in $\mathbf{J}$ ). This figure originally appeared in Tomchaney et al. (2014), which contains further information regarding experimental details. 


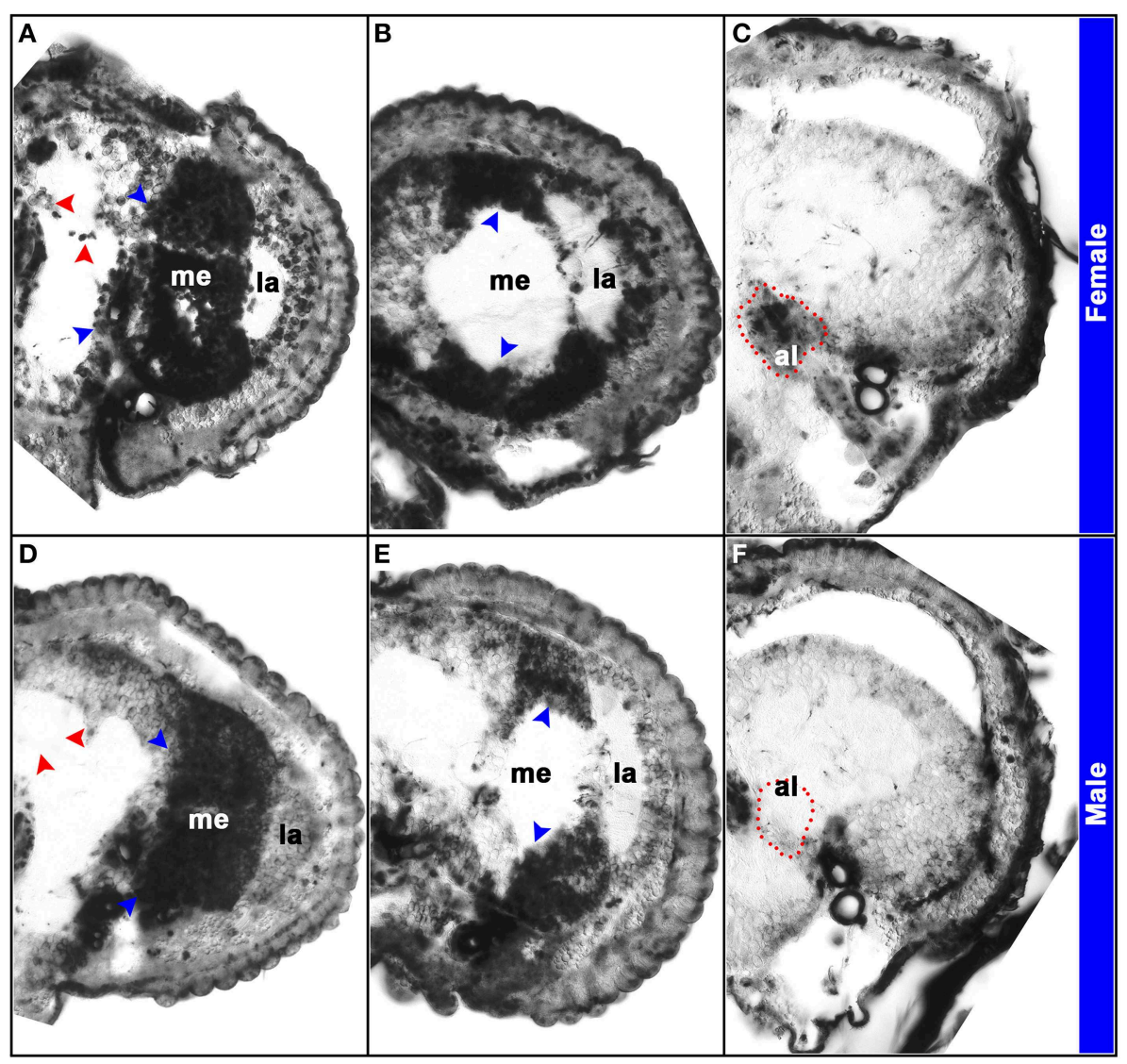

FIGURE 2 | Sex-specific expression patterns of $d s x$ in the $A$. aegypti pupal brain. Expression of $d s x$ was analyzed through in situ hybridization experiments performed on paraffin sections of female (A-C) and male (D-F) heads. Twelve micron sections through different portions of the brain revealed the antennal lobe (al), lamina (la), and medulla (me) in brain hemisegments oriented dorsal upward (A-F). Expression of $d s x$ is detected in the developing female and male visual systems (blue arrowheads in $\mathbf{A}, \mathbf{B}, \mathbf{D}, \mathbf{E})$. However, sex-specific expression of $d s x$ is detected in the antennal lobe (marked by red dots in $\mathbf{C , F}$ ) and mushroom bodies (red arrowheads in $\mathbf{A}, \mathbf{D}$ ). This figure originally appeared in Tomchaney et al. (2014), which provides further experimental details. terms linked to neurological processes or neural development, particularly the sensory system and sensory development, and 48 of which flank dimorphically expressed genes identified in the pupal head microarray experiments (Tomchaney et al., 2014). Together, these analyses support the hypothesis that Dsx is a regulator of sexually dimorphic gene expression in the A. aegypti nervous system and the development of sexually dimorphic traits in mosquitoes. This hypothesis was examined through functional genetic characterization of $d s x$ in A. aegypti.

\section{Functional Analysis of Sex-Specific Genes in the Mosquito Nervous System}

Small interfering RNAs (siRNAs), 20-25 nucleotide long doublestranded RNA (dsRNA) molecules that interfere with expression of genes complementary in nucleotide sequence, can be used to silence genes during $A$. aegypti development. In comparison to 400-600 bp dsRNAs, custom siRNAs are produced commercially en masse and are more readily designed to be both gene and species-specific. The time at which gene silencing initiates can be managed through control of siRNA delivery. This advantage facilitates the study of embryonic lethal genes during postembryonic stages of development; it also permits identification of the critical periods in which loss of gene function generates phenotypes of interest, information that may inform the design of control strategies (Clemons et al., 2010b; Zhang et al., 2015).

Microinjected siRNA (Clemons et al., 2010b) can be used to target $A$. aegypti developmental genes (Clemons et al., 2011; Haugen et al., 2011; Nguyen et al., 2013; Sarro et al., 2013; Tomchaney et al., 2014). siRNA can also be delivered to $A$. aegypti larvae via chitosan nanoparticles (Mysore et al., 2013, $2014 a, b)$ that are mixed with larval food and orally ingested by larvae, and which may promote the stability and cellular uptake of interfering RNA (Zhang et al., 2010). This technique, for which detailed methodology is available (Zhang et al., 2015), is relatively inexpensive, requires little equipment and labor, facilitates high-throughput analysis of multiple phenotypes including behavioral analyses (Zhang et al., 2010, 2015; Mysore 
et al., 2013, 2014a,b), and could likely be adapted for gene silencing studies in other insect species. Furthermore, chitosan, a non-toxic and biodegradable polymer (Dass and Choong, 2008), could potentially be utilized in the field.

siRNA-mediated silencing facilitated analysis of the function of $d s x$ during A. aegypti development. siRNAs corresponding to different target sequences in Aae $d s x$ exon 2, which is common to male and female splice variants (Salvemini et al., 2011), were injected into pupae (Tomchaney et al., 2014). The p53, synaptojanin, geko, rab6, and cyclin dependent kinase 4/6 genes are flanked by Dsx binding sites. The sex-specific pupal brain expression patterns of these genes were disrupted by silencing of $d s x$ (Figure 1), indicating that Dsx is required for sexually dimorphic gene expression in the developing $A$. aegypti CNS (Tomchaney et al., 2014). Analysis of the impact of developmental silencing of $d s x$ on adult phenotypes will facilitate analysis of adult female morphological, physiological, and behavioral characters that result from loss of $d s x$ function during A. aegypti development. In particular, it will be interesting to assess the impact of $d s x$ silencing on the structure and function of the olfactory system.

\section{Analysis of the A. aegypti Olfactory System}

Mosquitoes, including Aedes, show robust olfactory-driven behaviors, a number of which are sexually dimorphic (Bowen, 1992; Zwiebel and Takken, 2004; Carey and Carlson, 2011). Olfaction in adult A. aegypti is mediated by elaborate olfactory appendages, antennae, and maxillary palps that are adorned with many hair-like structures called sensilla. A great majority of these are sensory sensilla that house olfactory receptor neurons (ORNs) in which olfactory receptor (OR) proteins are embedded. A plethora of chemicals originating from blood meal host skin and breath, plant/nectar, and oviposition sites are detected by these ORNs (Bowen, 1992; Zwiebel and Takken, 2004). Olfaction initiates with interactions between specific odorants and distinct subpopulations of ORs present in the dendritic membrane of ORNs. While all the antennal segments of females are adorned with olfactory sensilla, they are present only on the terminal two segments in males. All types of olfactory sensilla in A. aegypti display sexual dimorphism in numbers. The most abundant type, trichodea sensilla that detect the majority of volatile cues derived from plants (in addition to host derived odorants), are four times more prevalent in females (Syed and Leal, 2009; Liu et al., 2013). Another category of sensilla, grooved pegs that primarily detect host-derived odors and express a distinct family of ionotropic receptors (IRs), are also at least twice as prevalent in females. Maxillary palps, the "broad spectrum odorant detectors" (Syed and Leal, 2007), have only one type of olfactory sensillum that is approximately twice more abundant in females (McIver, 1971). In absence of clear evidence in mosquitoes so far, it appears that sexually dimorphic behaviors potentially result from numerical differences in sensilla, and/or the relative proportion thereof, as has been recently reviewed for other blood-feeding insects (Syed, 2015). Sexual dimorphisms in the number and size of glomeruli in the antennal lobe of the $A$. aegypti brain have also been identified (Ignell et al., 2005).
It will be interesting to examine how developmental silencing of $d s x$ or other sex-determination genes impacts the sexspecific structure and function of the adult olfactory system and olfactory-driven behaviors in A. aegypti. For example, scanning electron microscopy could be used to explore resulting numerical and morphometric structural anomalies of the olfactory sensilla. Maxillary palp sensilla house three ORNs that respond to carbon dioxide, 1-octen-3-ol, and acetophenone respectively in Culex (Syed and Leal, 2007), Aedes (Grant and O'Connell, 1996), and Anopheles (Lu et al., 2007). To date, studies in all three species have been conducted exclusively in females, and it remains an exciting avenue to explore sexual differences, especially after $d s x$ manipulation, in males. Males are attracted to host odors, but likely differ from females in their response amplitude and dynamics to host chemostimuli. Sexual dimorphisms may particularly be expected at very close range and for landing responses, as well as in the male mating system which facilitates interception of females at the host (recently reviewed by Oliva et al., 2014). It is tempting to speculate that developmental differences will potentiate measureable neuroethological differences. A variety of behavioral assays can be employed to efficiently dissect the sexually dimorphic or isomorphic mosquito life behaviors mediated by odors: sugar feeding (Syed and Leal, 2008), host feeding (Sim et al., 2012), and oviposition (Laurence et al., 1985). It is predicted that loss of $d s x$ will disrupt some or all of these olfactory-driven behaviors that are critical to mosquito survival and reproduction. Ultimately, the overarching goal will be to identify and functionally characterize specific Dsx target genes that regulate sex-specific olfactory-driven behaviors.

\section{Future Functional Genetic Studies in $A$. aegypti}

Studies in D. melanogaster have demonstrated that Dsx and Fru function in the same neurons to establish neuronal wiring and behaviors (Rideout et al., 2007, 2010; Kimura et al., 2008). Neville et al. (2014) suggested that Drosophila Dsx and Fru act together, either in a physical complex or through co-regulation of target genes, to control sex-specific neural development. Although sex-specific Fru splice forms have been identified in A. aegypti (Salvemini et al., 2013), the expression patterns of these transcripts have not yet been assessed in the developing nervous system, and fru function, has not been characterized in mosquitoes. Given the likelihood of fertility defects in $d s x$ loss of function animals and the lack of marked balancer chromosomes in mosquitoes, conditional siRNA-mediated gene silencing has proven to be an excellent strategy for analysis of $d s x$ function, and this technique would likely permit analysis of fru function, as well as the functions of other components of the sex-specification pathway. The transcriptional targets of Dsx (Tomchaney et al., 2014) and Fru may also represent targets for vector control. It will also be interesting to characterize the functions of various ORs in males and females, particularly those that are known to be dimorphically expressed (Bohbot et al., 2007) and that may be direct or indirect targets of sex-specification genes. In 
addition to RNA interference, targeted mutagenesis is emerging as a viable option for assessing the function of these target genes.

Homing endonucleases, zinc-finger nucleases, and TALE nucleases (TALENs) have been used to generate heritable loss of function mutations in A. aegypti (Aryan et al., 2013a,b, 2014; Degennaro et al., 2013; Liesch et al., 2013; McMeniman et al., 2014). Degennaro et al. (2013) used zinc-finger nucleases to generate targeted mutations in the A. aegypti orco gene, which encodes the obligate co-receptor in the assembly and function of heteromeric OR/Orco complexes. Orco is crucial for discrimination between human vs. non-human hosts and for repulsion by volatile N,N-diethyl-meta-toluamide (DEET). Zinc-finger endonucleases were also used to target AaegGr3, which encodes a subunit of the heteromeric receptor complex required for carbon dioxide detection (McMeniman et al., 2014). CRISPR-Cas9 genome engineering was recently reported in A. aegypti (Basu et al., 2015; Dong et al., 2015; Kistler et al., 2015). This technology generates high levels of mutagenesis and is reportedly a cheaper, faster, and more flexible method for generating loss of function mutations. This technique, which is rapidly becoming the method of choice for mutagenesis studies in mosquitoes, will greatly facilitate interrogation of the adult A. aegypti olfactory system, olfactory development, and the development of sexually dimorphic traits in mosquitoes.

Despite substantial progress in mosquito genetic research, very few cis-regulatory elements (CREs), DNA sequences that control gene expression, have been identified in the mosquito genomes. This deficiency-a significant gap in basic knowledge of mosquito genetics-has resulted in a lack of drivers to manipulate or prevent gene expression in selected tissues at specific times. Such tools, which revolutionized research in genetic model organisms, would facilitate genetic studies and benefit all avenues of mosquito research, including analysis of neural development. Discovery of CRE drivers would also promote the development of transgenic insects for vector control, such as the female flightless mosquitoes generated with a flight muscle regulatory element (Fu et al., 2010; Wise De Valdez et al., 2011). FAIRE-seq, formaldehyde-assisted isolation of regulatory elements paired with DNA sequencing (Simon et al., 2012), a powerful new approach for global biochemical isolation of CREs through their lack of association with nucleosome proteins, will facilitate genome-wide discovery of putative A. aegypti CREs. Testing putative CREs in transgenic reporter assays will permit identification of gene drivers for the brain, olfactory system, and other tissues of vector importance. FAIRE-seq studies, as well as the use of other biochemical approaches (i.e., DNAse-seq)

\section{References}

Arensburger, P., Megy, K., Waterhouse, R. M., Abrudan, J., Amedeo, P., Antelo, B., et al. (2010). Sequencing of Culex quinquefasciatus establishes a platform for mosquito comparative genomics. Science 330, 86-88. doi: $10.1126 /$ science. 1191864 or computational approaches for the identification of insect CREs (Kazemian et al., 2014), will also facilitate analysis of gene regulatory networks in the developing nervous system. Moreover, since FAIRE assesses chromatin states, it is anticipated that FAIRE-seq might also be applied for epigenetic analysis of sexual dimorphism in $A$. aegypti, an exciting prospect.

\section{Conclusions}

Recent technical advances are facilitating functional genetic studies in $A$. aegypti, an emerging model for vector mosquito development. These techniques are being used to study the development of sexual dimorphism in neural tissues, particularly the brain and olfactory system. Comparison of female vs. male transcriptomes and detailed spatial analysis of gene expression patterns are uncovering sexual dimorphisms in the developing nervous system. siRNA-mediated gene silencing studies and targeted mutagenesis studies with emerging CRISPR/Cas9 technology can be used to assess the functional contributions of various genes to the development of sexual dimorphism. These studies are providing insight into the neurodevelopmental genetic basis for sexual dimorphism in vector mosquitoes and may promote the elucidation of novel genetic targets for vector control strategies.

\section{Author Contributions}

MS and ZS drafted this manuscript and are responsible for the summarization and interpretation of experiments described herein. They are accountable for the discussion provided in this review article, the final draft of which was approved by both authors.

\section{Acknowledgments}

Confocal images were acquired at the Indiana University School of Medicine, South Bend, IN Imaging and Flow Cytometry Core Facility. The dimorphism microarray study was funded by a University of Notre Dame Eck Institute for Global Health Pilot Study grant to MD. Optimization of siRNA-mediated gene targeting techniques in A. aegypti was supported by NIH/NIAID Award R01-AI081795 to MD. Analysis of $d s x$ was funded by a Bert Elwert Award in Medicine to MD. FAIREseq studies were supported by Indiana CTSI and Navari Family Foundation awards, and ongoing reporter analyses are funded by NIH/NIAID Award R21 AI117145-01 to MDS. Research in ZS laboratory is supported by a University of Notre Dame Eck Institute for Global Health Pilot Study grant. 
Aryan, A., Myles, K. M., and Adelman, Z. N. (2014). Targeted genome editing in Aedes aegypti using TALENs. Methods 69, 38-45. doi: 10.1016/j.ymeth.2014.02.008

Basu, S., Aryan, A., Overcash, J. M., Samuel, G. H., Anderson, M. A., Dahlem, T. J., et al. (2015). Proc. Natl. Acad. Sci. U.S.A. 112, 4038-4043. doi: $10.1073 /$ pnas. 1502370112

Bohbot, J., Pitts, R. J., Kwon, H. W., Rützler, M., Robertson, H. M., and Zwiebel, L. J. (2007). Molecular characterization of the Aedes aegypti odorant receptor gene family. Insect Mol. Biol. 16, 525-537. doi: 10.1111/j.1365-2583.2007.00748.x

Bowen, M. F. (1992). Terpene-sensitive receptors in female Culex pipiens mosquitos - electrophysiology and behavior. J. Insect Physiol. 38, 759-764. doi: 10.1016/0022-1910(92)90028-C

Burtis, K. C., and Baker, B. S. (1989). Drosophila doublesex gene controls somatic sexual differentiation by producing alternatively spliced mRNAs encoding related sex-specific polypeptides. Cell 56, 997-1010. doi: 10.1016/00928674(89)90633-8

Camara, N., Whitworth, C., and Van Doren, M. (2008). The creation of sexual dimorphism in the Drosophila soma. Curr. Top. Dev. Biol. 83, 65-107. doi: 10.1016/S0070-2153(08)00403-1

Carey, A. F., and Carlson, J. R. (2011). Insect olfaction from model systems to disease control. Proc. Natl. Acad. Sci. U.S.A. 108, 12987-12995. doi: 10.1073/pnas.1103472108

Christiansen, A. E., Keisman, E. L., Ahmad, S. M., and Baker, B. S. (2002). Sex comes in from the cold: the integration of sex and pattern. Trends Genet. 18, 510-516. doi: 10.1016/S0168-9525(02)02769-5

Clemons, A., Haugen, M., Flannery, E., Tomchaney, M., Kast, K., Jacowski, C., et al. (2010a). Aedes aegypti: an emerging model for vector mosquito development. Cold Spring Harb. Protoc. 2010:pdb.emo141. doi: 10.1101/pdb.emo141

Clemons, A., Haugen, M., Le, C., Mori, A., Tomchaney, M., Severson, D. W., et al. (2011). siRNA-mediated gene targeting in Aedes aegypti embryos reveals that frazzled regulates vector mosquito CNS development. PLoS ONE 6:e16730. doi: 10.1371/journal.pone.0016730

Clemons, A., Haugen, M., Severson, D., and Duman-Scheel, M. (2010b). Functional analysis of genes in Aedes aegypti embryos. Cold Spring Harb. Protoc. 2010:pdb.prot5511. doi: 10.1101/pdb.prot5511

Dass, C. R., and Choong, P. F. (2008). Chitosan-mediated orally delivered nucleic acids: a gutful of gene therapy. J. Drug Target. 16, 257-261. doi: $10.1080 / 10611860801900801$

Degennaro, M., McBride, C. S., Seeholzer, L., Nakagawa, T., Dennis, E. J., Goldman, C., et al. (2013). orco mutant mosquitoes lose strong preference for humans and are not repelled by volatile DEET. Nature 498, 487-491. doi: 10.1038 /nature 12206

Di Giovanni, S., Knights, C. D., Rao, M., Yakovlev, A., Beers, J., Catania, J., et al. (2006). The tumor suppressor protein p53 is required for neurite outgrowth and axon regeneration. EMBO J. 25, 4084-4096. doi: 10.1038/sj.emboj.7601292

Dong, S., Lin, J., Held, N. L., Clem, R. J., Passarelli, A. L., and Franz, A. W. (2015). Heritable CRISPR/Cas9-mediated genome editing in the yellow fever vector mosquito Aedes aegypti. PLoS ONE 10:e0122353. doi: 10.1371/journal.pone.0122353

Duman-Scheel, M., Pirkl, N., and Patel, N. H. (2002). Analysis of the expression pattern of Mysidium columbiae wingless provides evidence for conserved mesodermal and retinal patterning processes among insects and crustaceans. Dev. Genes. Evol. 212, 114-123. doi: 10.1007/s00427-002-0215-6

Flannery, E., VanZomeren-Dohm, A., Beach, P., Holland, W. S., and DumanScheel, M. (2010). Induction of cellular growth by the axon guidance regulators netrin A and semaphorin-1a. Dev. Neurobiol. 70, 473-484. doi: 10.1002/dneu.20788

Fu, G., Lees, R. S., Nimmo, D., Aw, D., Jin, L., Gray, P., et al. (2010). Femalespecific flightless phenotype for mosquito control. Proc. Natl. Acad. Sci. U.S.A. 107, 4550-4554. doi: 10.1073/pnas.1000251107

Grant, A. J., and O'Connell, R. J. (1996). Electrophysiological responses from receptor neurons in mosquito maxillary palp sensilla. Ciba Found. Symp. 200, 233-248.

Haugen, M., Flannery, E., Tomchaney, M., Mori, A., Behura, S. K., Severson, D. W., et al. (2011). Semaphorin-1a is required for Aedes aegypti embryonic nerve cord development. PLOS ONE 6:e21694. doi: 10.1371/journal.pone.0021694

Haugen, M., Tomchaney, M., Kast, K., Flannery, E., Clemons, A., Jacowski, C., et al. (2010). Whole-mount in situ hybridization for analysis of gene expression during Aedes aegypti development. Cold Spring. Harb. Protoc 2010:pdb.prot5509. doi: 10.1101/pdb.prot5509

Holt, R. A., Subramanian, G. M., Halpern, A., Sutton, G. G., Charlab, R., Nusskern, D. R., et al. (2002). The genome sequence of the malaria mosquito Anopheles gambiae. Science 298, 129-149. doi: 10.1126/science.1076181

Ignell, R., Dekker, T., Ghaninia, M., and Hansson, B. S. (2005). Neuronal architecture of the mosquito deutocerebrum. J. Comp. Neurol. 493, 207-240. doi: $10.1002 /$ cne. 20800

Kazemian, M., Suryamohan, K., Chen, J. Y., Zhang, Y., Samee, M. A., Halfon, M. S., et al. (2014). Evidence for deep regulatory similarities in early developmental programs across highly diverged insects. Genome Biol. Evol. 6, 2301-2320. doi: $10.1093 /$ gbe/evu184

Kimura, K. (2011). Role of cell death in the formation of sexual dimorphism in the Drosophila central nervous system. Dev. Growth Differ. 53, 236-244. doi: 10.1111/j.1440-169X.2010.01223.x

Kimura, K., Hachiya, T., Koganezawa, M., Tazawa, T., and Yamamoto, D. (2008). Fruitless and doublesex coordinate to generate male-specific neurons that can initiate courtship. Neuron 59, 759-769. doi: 10.1016/j.neuron.2008.06.007

Kimura, K., Ote, M., Tazawa, T., and Yamamoto, D. (2005). Fruitless specifies sexually dimorphic neural circuitry in the Drosophila brain. Nature 438, 229-233. doi: 10.1038/nature04229

Kistler, K. E., Vosshall, L. B., and Matthews, B. J. (2015). Genome-engineering with CRISPR-Cas9 in the mosquito Aedes aegypti. Cell Rep. 11, 51-60. doi: 10.1016/j.celrep.2015.03.009

Laurence, B. R., Mori, K., Otsuka, T., Pickett, J. A., and Wadhams, L. J. (1985). Absolute-configuration of mosquito oviposition attractant pheromone, 6-acetoxy-5-hexadecanolide. J. Chem. Ecol. 11, 643-648. doi: 10.1007/BF00988573

Lee, G., Hall, J. C., and Park, J. H. (2002). Doublesex gene expression in the central nervous system of Drosophila melanogaster. J. Neurogenet. 16, 229-248. doi: 10.1080/01677060216292

Liesch, J., Bellani, L. L., and Vosshall, L. B. (2013). Functional and genetic characterization of neuropeptide Y-like receptors in Aedes aegypti. PLoS Negl. Trop. Dis. 7:e2486. doi: 10.1371/journal.pntd.0002486

Liu, F., Chen, L., Appel, A. G., and Liu, N. (2013). Olfactory responses of the antennal trichoid sensilla to chemical repellents in the mosquito, Culex quinquefasciatus. J. Insect Physiol. 59, 1169-1177. doi: 10.1016/j.jinsphys.2013.08.016

Lu, T., Qiu, Y. T., Wang, G., Kwon, J. Y., Rutzler, M., Kwon, H. W., et al. (2007). Odor coding in the maxillary palp of the malaria vector mosquito Anopheles gambiae. Curr. Biol. 17, 1533-1544. doi: 10.1016/j.cub.2007.07.062

Magnusson, K., Mendes, A. M., Windbichler, N., Papathanos, P. A., Nolan, T., Dottorini, T., et al. (2011). Transcription regulation of sex-biased genes during ontogeny in the malaria vector Anopheles gambiae. PLoS ONE 6:e21572. doi: 10.1371/journal.pone.0021572

McIver, S. (1971). Comparative studies on sense organs on antennae and maxillary palps of selected male Culicine mosquitoes. Can. J. Zool. 49, 235-239. doi: 10.1139/z71-033

McMeniman, C. J., Corfas, R. A., Matthews, B. J., Ritchie, S. A., and Vosshall, L. B. (2014). Multimodal integration of carbon dioxide and other sensory cues drives mosquito attraction to humans. Cell 156, 1060-1071. doi: 10.1016/j.cell.2013.12.044

Mellert, D. J., Knapp, J. M., Manoli, D. S., Meissner, G. W., and Baker, B. S. (2010). Midline crossing by gustatory receptor neuron axons is regulated by fruitless, doublesex and the roundabout receptors. Development 137, 323-332. doi: $10.1242 /$ dev.045047

Mysore, K., Andrews, E., Li, P., and Duman-Scheel, M. (2014a). Chitosan/siRNA nanoparticle targeting demonstrates a requirement for single-minded during larval and pupal olfactory system development of the vector mosquito Aedes aegypti. BMC Dev. Biol. 14:9. doi: 10.1186/1471-213X-14-9

Mysore, K., Flannery, E., Leming, M. T., Tomchaney, M., Shi, L., Sun, L., et al. (2014b). Role of semaphorin-1a in the developing visual system of the disease vector mosquito Aedes aegypti. Dev. Dyn. 243, 1457-1469. doi: 10.1002/dvdy. 24168

Mysore, K., Flannery, E. M., Tomchaney, M., Severson, D. W., and Duman-Scheel, M. (2013). Disruption of Aedes aegypti olfactory system development through chitosan/siRNA nanoparticle targeting of semaphorin-1a. PLoS Negl. Trop. Dis. 7:e2215. doi: 10.1371/journal.pntd.0002215 
Mysore, K., Flister, S., Muller, P., Rodrigues, V., and Reichert, H. (2011). Brain development in the yellow fever mosquito Aedes aegypti: a comparative immunocytochemical analysis using cross-reacting antibodies from Drosophila melanogaster. Dev. Genes Evol. 221, 281-296. doi: 10.1007/s00427-011-0376-2

Neafsey, D. E., Waterhouse, R. M., Abai, M. R., Aganezov, S. S., Alekseyev, M. A., Allen, J. E., et al. (2015). Mosquito genomics. Highly evolvable malaria vectors: the genomes of 16 Anopheles mosquitoes. Science 347:1258522. doi: $10.1126 /$ science. 1258522

Nene, V., Wortman, J. R., Lawson, D., Haas, B., Kodira, C., Tu, Z. J., et al. (2007). Genome sequence of Aedes aegypti, a major arbovirus vector. Science 316, 1718-1723. doi: 10.1126/science. 1138878

Neville, M. C., Nojima, T., Ashley, E., Parker, D. J., Walker, J., Southall, T., et al. (2014). Male-specific fruitless isoforms target neurodevelopmental genes to specify a sexually dimorphic nervous system. Curr. Biol. 24, 229-241. doi: 10.1016/j.cub.2013.11.035

Nguyen, C., Andrews, E., Le, C., Sun, L., Annan, Z., Clemons, A., et al. (2013). Functional genetic characterization of salivary gland development in Aedes aegypti. Evodevo 4:9. doi: 10.1186/2041-9139-4-9

Oliva, C. F., Damiens, D., and Benedict, M. Q. (2014). Male reproductive biology of Aedes mosquitoes. Acta Trop. 132S, S12-S19. doi: 10.1016/j.actatropica.2013.11.021

Patel, N. H., Hayward, D. C., Lall, S., Pirkl, N. R., Dipietro, D., and Ball, E. E. (2001). Grasshopper hunchback expression reveals conserved and novel aspects of axis formation and segmentation. Development 128, 3459-3472.

Rideout, E. J., Billeter, J. C., and Goodwin, S. F. (2007). The sex-determination genes fruitless and doublesex specify a neural substrate required for courtship song. Curr. Biol. 17, 1473-1478. doi: 10.1016/j.cub.2007.07.047

Rideout, E. J., Dornan, A. J., Neville, M. C., Eadie, S., and Goodwin, S. F. (2010). Control of sexual differentiation and behavior by the doublesex gene in Drosophila melanogaster. Nat. Neurosci. 13, 458-466. doi: 10.1038/nn.2515

Ryner, L. C., Goodwin, S. F., Castrillon, D. H., Anand, A., Villella, A., Baker, B. S., et al. (1996). Control of male sexual behavior and sexual orientation in Drosophila by the fruitless gene. Cell 87, 1079-1089. doi: 10.1016/S00928674(00)81802-4

Salvemini, M., D’Amato, R., Petrella, V., Aceto, S., Nimmo, D., Neira, M., et al. (2013). The orthologue of the fruitfly sex behaviour gene fruitless in the mosquito Aedes aegypti: evolution of genomic organisation and alternative splicing. PLoS ONE 8:e48554. doi: 10.1371/journal.pone.0048554

Salvemini, M., Mauro, U., Lombardo, F., Milano, A., Zazzaro, V., Arca, B., et al. (2011). Genomic organization and splicing evolution of the doublesex gene, a Drosophila regulator of sexual differentiation, in the dengue and yellow fever mosquito Aedes aegypti. BMC Evol. Biol. 11:41. doi: 10.1186/1471-2148-11-41

Sarro, J., Andrews, E., Sun, L., Behura, S. K., Tan, J. C., Zeng, E., et al. (2013). Requirement for commissureless 2 function during dipteran insect nerve cord development. Dev. Dyn. 242, 1466-1477. doi: 10.1002/dvdy.24059

Shiraiwa, T., Nitasaka, E., and Yamazaki, T. (2000). Geko, a novel gene involved in olfaction in Drosophila melanogaster. J. Neurogenet. 14, 145-164. doi: 10.3109/01677060009083480

Sim, S., Ramirez, J. L., and Dimopoulos, G. (2012). Dengue virus infection of the Aedes aegypti salivary gland and chemosensory apparatus induces genes that modulate infection and blood-feeding behavior. PLoS Pathog. 8:e1002631. doi: 10.1371/journal.ppat.1002631

Simon, J. M., Giresi, P. G., Davis, I. J., and Lieb, J. D. (2012). Using formaldehydeassisted isolation of regulatory elements (FAIRE) to isolate active regulatory DNA. Nat. Protoc. 7, 256-267. doi: 10.1038/nprot.2011.444

Sutcliffe, J. E., Korenjak, M., and Brehm, A. (2003). Tumour suppressors-a fly's perspective. Eur. J. Cancer 39, 1355-1362. doi: 10.1016/S0959-8049(03)00263-6

Syed, Z. (2015). Chemical ecology and olfaction in arthropod vectors of diseases. Curr. Opin. Insect Sci. 10, 83-89. doi: 10.1016/j.cois.2015.04.011

Syed, Z., and Leal, W. S. (2007). Maxillary palps are broad spectrum odorant detectors in Culex quinquefasciatus. Chem. Senses 32, 727-738. doi: 10.1093/chemse/bjm040

Syed, Z., and Leal, W. S. (2008). Mosquitoes smell and avoid the insect repellent DEET. Proc. Natl. Acad. Sci. U.S.A. 105, 13598-13603. doi: 10.1073/pnas.0805312105

Syed, Z., and Leal, W. S. (2009). Acute olfactory response of Culex mosquitoes to a human- and bird-derived attractant. Proc. Natl. Acad. Sci. U.S.A. 106, 18803-18808. doi: 10.1073/pnas.0906932106

Tomchaney, M., Mysore, K., Sun, L. H., Li, P., Emrich, S. J., Severson, D. W., et al. (2014). Examination of the genetic basis for sexual dimorphism in the Aedes aegypti (dengue vector mosquito) pupal brain. Biol. Sex Diff. 5:10. doi: 10.1186/s13293-014-0010-x

Verstreken, P., Koh, T. W., Schulze, K. L., Zhai, R. G., Hiesinger, P. R., Zhou, Y., et al. (2003). Synaptojanin is recruited by endophilin to promote synaptic vesicle uncoating. Neuron 40, 733-748. doi: 10.1016/S0896-6273(03) 00644-5

Wise De Valdez, M. R., Nimmo, D., Betz, J., Gong, H. F., James, A. A., Alphey, L., et al. (2011). Genetic elimination of dengue vector mosquitoes. Proc. Natl. Acad. Sci. U.S.A. 108, 4772-4775. doi: 10.1073/pnas.1019295108

Zhang, X., Mysore, K., Flannery, E., Michel, K., Severson, D. W., Zhu, K. Y., et al. (2015). Chitosan/interfering RNA nanoparticle mediated gene silencing in disease vector mosquito larvae. J. Vis. Exp. 97:e52523. doi: 10.3791/52523

Zhang, X., Zhang, J., and Zhu, K. Y. (2010). Chitosan/double-stranded RNA nanoparticle-mediated RNA interference to silence chitin synthase genes through larval feeding in the African malaria mosquito (Anopheles gambiae). Insect Mol. Biol. 19, 683-693. doi: 10.1111/j.1365-2583.2010.01029.x

Zwiebel, L. J., and Takken, W. (2004). Olfactory regulation of mosquitohost interactions. Insect Biochem. Mol. Biol. 34, 645-652. doi: 10.1016/j.ibmb.2004.03.017

Conflict of Interest Statement: The authors declare that the research was conducted in the absence of any commercial or financial relationships that could be construed as a potential conflict of interest.

Copyright (C) 2015 Duman-Scheel and Syed. This is an open-access article distributed under the terms of the Creative Commons Attribution License (CC BY). The use, distribution or reproduction in other forums is permitted, provided the original author(s) or licensor are credited and that the original publication in this journal is cited, in accordance with accepted academic practice. No use, distribution or reproduction is permitted which does not comply with these terms. 